\title{
Comparación de los resultados en la resolución de ambigüedades para una red de estaciones GNSS en Europa con el uso de datos GPS y la combinación con datos GLONASS, utilizando el programa de procesamiento Bernese
}

\author{
Comparison of the Results in the Ambiguity Resolution for a Network of GNSS \\ Stations in Europe Using GPS Data and the Combination with GLONASS Data, \\ Using the Bernese GNSS Processing Software
}

\author{
Diana Paniagua-Jiménez \\ diananinette21@gmail.com \\ Escuela de Topografía, Catastro y Geodesia \\ Universidad Nacional \\ Heredia, Costa Rica \\ Jose Valverde-Calderón \\ jose.valverde.calderon@una.cr \\ Escuela de Topografía, Catastro y Geodesia \\ Universidad Nacional \\ Heredia, Costa Rica
}

Recibido-Received: 2/may/2016 / Corregido-Corrected: 16/jun /2016.

Aceptado-Accepted: 29/jun/2016 / Publicado-Published: 31/jul /2017.

\begin{abstract}
Resumen
Los parámetros de la ambigüedad en las ecuaciones de observación originales son números enteros. Técnicamente son necesarios dos pasos para fijar las ambigüedades: primero, los parámetros son estimados como valores reales; en el segundo paso se fijan a números enteros. La fijación de las ambigüedades disminuye el número de parámetros a estimar en el ajuste final, lo cual lleva a un ahorro en número de elementos que se almacena en la memoria, en el tiempo invertido en el procesamiento y una mejora en la calidad del cálculo de las coordenadas. Se efectuó la comparación entre los resultados de dos procesamientos de la misma red: en el primero, se procesaron solo observaciones GPS; en el segundo cálculo, se procesaron de forma conjunta observaciones GPS y GLONASS. La red usada en el proyecto está conformada por 25 estaciones GNSS, ubicadas en Europa. El procesamiento se efectuó con el programa Bernese 5.2. Se concluyó que la selección de la estrategia para fijar ambigüedades es un aspecto que debe analizarse detenidamente, debido al impacto que tiene el método de cálculo seleccionado sobre los resultados finales y el tiempo de procesamiento requerido.
\end{abstract}

Palabras claves: Geodesia; Bernese; GPS; GLONASS; ambigüedades.

Uniciencia es una revista de acceso abierto/ Uniciencia is an Open Access Journal. 
UNICIENCIA Vol. 31, No. 2, pp. 1-17. Julio-diciembre, 2017.

ISSN Electrónico: 2215-3470

URL: www.revistas.una.ac.cr/uniciencia

DOI: http://dx.doi.org/10.15359/ru.31-2.1

Email: revistauniciencia@una.cr

\begin{abstract}
The parameters of the ambiguity in the original observation equations are integer numbers. Technically two procedures are necessary to fix the ambiguities: first, the parameters are estimated as real values; in the second step, they are set to integers. Fixing the ambiguities decreases the number of parameters to be estimated in the final adjustment of the network, taking this result in a reduction in the number of items stored in memory, in the time investing in the processing and an improvement in the quality of the calculate of coordinates. It was made the comparative analysis between the results after processing the network two times: in the first one, only GPS observation was processed; in the second one, both GPS and GLONASS observations were processed. The network used in the project has 25 GNSS stations, located in Europe. The processing was made using the Bernese GNSS software version 5.2. The main conclusion is the importance in the selection of the ambiguity strategy due to is a topic that require an complete analysis, due to the impact that the choose computation method have in the finals results and in the processing time.
\end{abstract}

Keywords: Geodesy; Bernese; GPS; GLONASS; ambiguity

El Servicio GNSS Internacional (IGS, por sus siglas en inglés) es el encargado de adquirir, recopilar, distribuir, procesar y archivar los datos de observaciones de los sistemas satelitales de navegación global (GNSS, por sus siglas en inglés). Actualmente el IGS procesa datos del sistema de posicionamiento global (GPS, por sus siglas en inglés), mantenido por el Departamento de Defensa de Estados Unidos, del Sistema Satelital de Navegación Global (GLONASS, por sus siglas en ruso), el cual es operado por la Federación Rusa y otros sistemas de navegación por satélite. Como resultado se generan productos tales como efemérides precisas, información de parámetros troposféricos, coordenadas de la red del IGS, entre otros (Dach, Lutz, Walser \& Fridez, 2015). El IGS fue aceptado en 1993 por la Asociación Internacional de Geodesia (IAG, por sus siglas en inglés) y comenzó a operar el 1 de enero de 1994, inicialmente brindó datos y órbitas GPS (IGS Central Bureau Information System, 2004).

Con el fin de evaluar la posibilidad de incorporar los datos de sistema GLONASS, el IGS desarrolló el experimento GLONASS Internacional (IGEX-98, por sus siglas en inglés) el cual finalizó en el año 2000. En el marco de desarrollo del IGEX-98, se estableció una red mundial de receptores GNSS (entendiéndose como receptor GNSS aquel que recibe y registra las señales de al menos dos sistemas de navegación por satélite) que registraron datos por un periodo mínimo de tres meses. Los principales resultados fueron órbitas precisas GLONASS con una exactitud promedio de $20 \mathrm{~cm}$ en el componente radial, posiciones de las estaciones con una exactitud centimétrica y parámetros de transformación entre el sistema de referencia del sistema GPS y el de GLONASS (Willis et al., 1999)

Con la creciente disponibilidad de receptores GNSS, la revitalización del sistema ruso, dada principalmente por el aumento de satélites en su constelación, y la publicación de órbitas para este mismo por parte del por el IGS, resulta una tarea importante estudiar el uso de los datos GLONASS y su aporte al mantenimiento de los marcos de referencia, particularmente cuando los datos son procesados de forma conjunta con datos GPS (Bruyninx, 2006).

En el 2006, en el Observatorio Real de Bélgica se realizó una investigación con el fin de estudiar las ventajas y desventajas del análisis combinado con datos de los sistemas GPS y GLONASS, para una red regional que usa el programa Bernese v5.0. Luego del procesamiento, los resultados mostraron diferencias entre las coordenadas con datos solamente GPS y las 
coordenadas con datos GPS y GLONASS en un nivel de 1-2 mm en la componente horizontal y entre $2 \mathrm{~mm}$ y $6 \mathrm{~mm}$ en la vertical. En su momento, la causa de las discrepancias no fue clara y se recomendó efectuar más estudios (Bruyninx, 2006).

La Subcomisión 1.3a de la IAG, a cargo del Marco de Referencia para Europa (EUREF, por sus siglas en inglés) concluyó que al procesar solo datos GPS y luego usar datos combinados GPS y GLONASS, con el programa Bernese V5.0, las coordenadas pueden variar en los componentes horizontales entre 1 y $2 \mathrm{~mm}$ y en la vertical hasta $6 \mathrm{~mm}$ al momento de comparar las soluciones (Habrich, 2009).

La resolución N. 3 del 10 de agosto de 2011 del Sistema de Referencia Geocéntrico para las Américas (SIRGAS) crea el Proyecto SIRGAS-GLONASS, en el cual se efectuaron estudios para analizar la conveniencia del uso de la técnica satelital GLONASS en la generación de productos asociados a SIRGAS; Cioce, Robin, Mateo \& Mackern (2013) demostraron que los resultados del procesamiento combinado de datos GPS y GLONASS en el programa Bernese versión 5.0 tiene la tendencia a ser menos preciso que los del procesamiento con solamente datos GPS, situación reportada en Bruyninx (2006) y Habrich (2009).

En agosto de 2013 se publicó la versión 5.2 del programa Bernese, el cual, entre sus características, de acuerdo con Dach \& Walser (2013), tiene una mejora en el tratamiento conjunto de datos GPS y GLONASS. Por tanto, la presente investigación tiene como objeto determinar si en la actualidad el producto del procesamiento conjunto GPS y GLONASS es mejor o al menos semejante a los resultados obtenidos con únicamente datos GPS, con enfoque en el tema de la resolución de ambigüedades.

En general, los receptores GNSS reciben dos tipos de señales (observables): código y fase de la portadora. La fase de la portadora es una medición de la fase de la señal satelital recibida relativa a la portadora generada por el receptor en un tiempo t; el número completo de ondas entre el receptor y el satélite no puede ser contabilizado en el momento inicial en que el receptor empieza a registrar. Por lo tanto, la medición de la fase de la portadora es la medición de la fase fraccional $(\underline{\mathrm{Xu}, 2007})$.

La parte fraccional de la portadora puede ser medida con una precisión mejor al $1 \%$ de la longitud de onda, lo cual corresponde a una precisión milimétrica. Esta es la razón del porqué las mediciones de fase son más precisas que las mediciones de código. Una onda completa de la portadora es llamada un ciclo; el número ambiguo entero de ciclos en la medición de fase es llamado ambigüedad y como se comentó es desconocida al momento de iniciar una medición ( $\underline{\mathrm{Xu}, 2007})$.

La ambigüedad inherente a la medición de fase depende solo del receptor y del satélite, por lo que no hay una dependencia en el tiempo, mientras el registro de datos sea mantenido sin interrupción. Inicialmente las ambigüedades son estimadas como números reales, sin embargo, para incrementar la calidad de la solución es necesario estimarlas de acuerdo con su naturaleza, la cual es que corresponden a números enteros. Así, tan pronto la ambigüedad es determinada como un número entero, se dice que esta ha sido resuelta o fijada (Hofmmann-Wellenhof, Lichtenegger \& Wasle, 2008).

El acceso a la naturaleza entera de la ambigüedad es difícil debido a la influencia provocada por los retrasos del hardware en el receptor y el desplazamiento de la fase inicial entre el receptor y el satélite (Dach et al., 2015). El uso de dobles diferencias en el procesamiento es importante; la razón es que en el caso de simples diferencias un parámetro desconocido adicional para el reloj del 
UNICIENCIA Vol. 31, No. 2, pp. 1-17. Julio-diciembre, 2017.

receptor debe ser considerado, lo cual evita una efectiva separación de las ambigüedades enteras y el error del reloj del receptor. Cuando se aplican dobles diferencias el término del reloj del receptor es eliminado por lo que "aislar" la ambigüedad es posible (Hofmmann-Wellenhof et al., 2008).

Con base en lo comentado, es un tema fundamental fijar las ambigüedades, por lo que la estrategia seleccionada para esto incide en la calidad del procesamiento. Al momento de escoger el método para resolverlas, se deben tomar en cuenta parámetros como: la longitud de las líneas base, los tiempos de ocupación en cada estación, los observables disponibles y los sistemas GNSS observados.

De acuerdo con Mervart (1995), se han propuesto numerosos métodos para tratar con la resolución de parámetros iniciales de la ambigüedad de fase. Se distinguen dos casos:

1. Posicionamiento estático clásico: El tiempo de ocupación en el sitio es largo (horas a días), el número de mediciones es grande. Esto implica que en las líneas base cortas, los errores medios cuadráticos de las ambigüedades estimadas sean menores que $1 \mathrm{~Hz}$.

2. Posicionamiento estático rápido: El tiempo de ocupación del sitio es corto (varios minutos), el error medio cuadrático de los valores correctos de las ambigüedades estimadas es del orden de $1 \mathrm{~Hz}$ o aún mayor.

El software de procesamiento Bernese tiene cuatro estrategias para la resolución de ambigüedades las cuales son: ROUND, SIGMA, SEARCH y QIF (Dach et al., 2015). Se describen, a continuación, cada una de esas estrategias.

Estrategia ROUND. Es la estrategia de resolución de ambigüedad más simple, redondea la estimación de valor real al entero más cercano, sin utilizar ninguna información de varianzacovarianza. No se recomienda utilizar la estrategia ROUND sobre las líneas de base largas, solo de unos pocos kilómetros (Dach et al., 2015).

1. Estrategia SEARCH. La estrategia SEARCH está relacionada al algoritmo llamado FARA (Dach et al., 2015). Las características principales de uso del algoritmo FARA son (Hofmmann-Wellenhof et al., 2008):

2. Usa información estadística del ajuste inicial con el fin de seleccionar el rango de búsqueda.

3. Usa información de la matriz de varianza-covarianza para rechazar conjuntos de ambigüedades que no son aceptables desde el punto de vista estadístico.

4. Aplica pruebas de hipótesis para seleccionar el conjunto de ambigüedades enteras que ofrece el mejor resultado.

Si ambas frecuencias están disponibles (mediciones L1 y L2 procesadas) usualmente varios minutos de datos son suficientes para resolver las ambigüedades y lograr una precisión de alrededor de un centímetro (Dach et al., 2015). 
ISSN Electrónico: 2215-3470

DOI: http://dx.doi.org/10.15359/ru.31-2.1
UNICIENCIA Vol. 31, No. 2, pp. 1-17. Julio-diciembre, 2017. URL: www.revistas.una.ac.cr/uniciencia Email: revistauniciencia@una.cr

Estrategia SIGMA. Los errores medios cuadráticos de cada posible ambigüedad de dobles diferencias son ordenados de forma ascendente. Dentro de cada iteración, las ambigüedades mejor determinadas, considerando el parámetro Nmax (correspondiente con el número máximo a fijar por ciclo de cálculo), son luego resueltas (redondeadas al entero más cercano) (Dach et al., 2015).

Se recomienda utilizar esta estrategia en dos casos (Dach et al., 2015):

1. Solamente si mediciones de una frecuencia son procesadas, pero la sesión tiene un tiempo de duración amplio (varias horas). Las líneas base no deberán ser muy extensas (menos de $20 \mathrm{~km})$.

2. Mediciones de código de alta calidad están disponibles en ambas frecuencias. En este caso es posible utilizar la combinación lineal Melbourne-Wübbena (L6). Las líneas base pueden ser muy largas (superior a varios cientos de kilómetros). Las sesiones de medición también deberán ser largas (varias horas).

Estrategia QIF. Para la estrategia Quasi Ionosphere-Free (QIF, por sus siglas en inglés) tanto L1 y L2 son requeridas como observaciones. El algoritmo QIF se utiliza para resolver las ambigüedades de líneas hasta $2000 \mathrm{~km}$ de largo (Dach et al., 2015).

El ajuste inicial por mínimos cuadrados utilizando ambas frecuencias L1 y L2 da como resultado la estimación del valor real de la ambigüedad y, además, se calculan los correspondientes bias libres de ionósfera; este sesgo puede ser expresado en ciclos de narrow-lane (un ciclo corresponde a la longitud de onda $\left.\lambda_{3}=c /\left(f_{1}+f_{2}\right) \approx 11 \mathrm{~cm}\right)($ Dach at al., 2015).

Para las líneas base largas (más largas que $10 \mathrm{~km}$ ), el procesamiento separado de dos frecuencias L1 y L2 no da suficientes valores reales estimados de $b_{1}$ y $b_{2}$ debido a la influencia de la refracción ionosférica (Dach at al., 2015). Se consideran dos tipos de modelos para reducir el biases ionosféricos:

El primer modelo consiste en una corrección ionosférica apriori estimada para el satélite i, receptor $\mathrm{k}$ y época tj. La estimación de los parámetros de corrección sin ninguna restricción será equivalente al procesamiento de la combinación lineal libre de ionósfera. Si se quieren resolver los números enteros de las ambigüedades, es necesario restringirlos dentro de unos cuantos decímetros. Estas restricciones pueden ser logradas introduciendo una observación artificial con un peso preliminar diferente de cero (Dach et al., 2015).

El segundo es el modelo determinista, el cual considera la ionósfera como una capa simple. Esta se considera a una altura alrededor de $350 \mathrm{~km}$ sobre la superficie de la Tierra con espesor infinitesimal. El modelado se da en series de coeficientes armónicos en latitud y ángulo horario del Sol. Este deberá ser utilizado si se procesan líneas bases largas (Dach et al., 2015).

En la tabla 1 se aprecian las características de las diferentes estrategias para resolver ambigüedades. 
UNICIENCIA Vol. 31, No. 2, pp. 1-17. Julio-diciembre, 2017.

Email: revistauniciencia@una.cr

Tabla 1

Características de las estrategias del software Bernese

\begin{tabular}{l|c|l}
\hline Estrategias & Longitud de línea base & \multicolumn{1}{c}{ Combinación lineal } \\
\cline { 2 - 3 } & $<40 \mathrm{~km}$ & \multicolumn{1}{c}{ L1 \& L2 } \\
\cline { 2 - 3 } SIGMA & $<200 \mathrm{~km}$ & $\begin{array}{l}\text { Wide-lane: L5, introduciendo coordenadas, parámetros troposféricos } \\
\text { y un modelo ionosféricos. } \\
\text { Narrow-lane: introduciendo ambigüedades wide-lane y estimando } \\
\text { coordenadas y parámetros troposféricos. }\end{array}$ \\
\cline { 2 - 3 } & $<6000 \mathrm{~km}$ & $\begin{array}{l}\text { Wide-lane: MELWUEBB, introduciendo sesgo de código diferencial } \\
\text { (DCB). } \\
\text { Narrow-lane: introduciendo ambigüedades wide-lane y estimando } \\
\text { coordenadas y parámetros troposféricos. }\end{array}$ \\
\hline SEARCH & $<10 \mathrm{~km}$ & \multicolumn{1}{c}{ L1 \& L2 } \\
\hline ROUND & Bases cortas & L1 \& L2 \\
\hline QIF & $<2000 \mathrm{~km}$ & \multicolumn{1}{c}{} \\
\hline
\end{tabular}

Nota: Elaboración propia, a partir de la información encontrada en Dach et al. (2015).

\section{Consideraciones para resolver ambigüedades en Bernese}

Es importante conocer cuáles son las consideraciones que se deben tomar en cuenta a la hora de resolver ambigüedades con el software Bernese. Si ambas frecuencias están disponibles, se tiene las siguientes opciones (Dach et al., 2015):

1. Para las líneas base muy cortas, las ambigüedades pueden resolverse de forma independiente en L1 y L2 con el algoritmo de SIGMA. Si se procesan datos de frecuencia simple, no hay muchas posibilidades para resolverlas, pero se puede utilizar el algoritmo SEARCH (para sesiones cortas) o SIGMA (para las sesiones largas).

2. Para las líneas bases más extensas (hasta unos $2000 \mathrm{~km}$ ) es posible procesar las portadoras juntas y tratar de resolver ambas ambigüedades (L1 y L2) en el mismo procesamiento. Las estrategias recomendadas son QIF para las sesiones largas y la SEARCH para mediciones y líneas de base cortas.

3. Usando la técnica Wide Lane cada línea base se procesa dos veces; usando solamente observaciones de fase, se obtienen resultados satisfactorios para vectores de $100 \mathrm{~km}$ o más largas si un buen modelo ionosférico a priori es utilizado. En la subsiguiente ejecución la combinación lineal L3 es procesada, las ambigüedades Wide Lane son introducidas como conocidas y las Narrow Lane son resueltas. Es posible utilizar dos combinaciones lineales en una portadora, pero la resolución de la ambigüedad se lleva a cabo de forma independiente en ambas señales. 
ISSN Electrónico: 2215-3470

DOI: http://dx.doi.org/10.15359/ru.31-2.1
UNICIENCIA Vol. 31, No. 2, pp. 1-17. Julio-diciembre, 2017. URL: www.revistas.una.ac.cr/uniciencia Email: revistauniciencia@una.cr

\section{Metodología}

Para efectuar la investigación, se definió una red con 25 estaciones GNSS de medición continua ubicadas en Europa (ver figura 1). El procesamiento se desarrolló en dos etapas: el primero cubre un periodo de 3 semanas del año 2014, que inicia en la semana GPS 1774 hasta la semana 1776; en detalle el día 005, el cual corresponde al 5 de enero del 2014, hasta el día 025 el cual corresponde al 25 de enero del 2014. Esta etapa se desarrolló para efectuar pruebas con las distintas opciones de procesamiento que ofrece el programa y, a partir de acá, desarrollar la etapa 2, la cual consistió en procesar 26 semanas del año 2014, se inició en la semana GPS 1774 hasta la 1798; en detalle, el día 005, el cual corresponde al 5 de enero del 2014, hasta el 186 (5 de julio del 2014). La estrategia de cálculo usada en la etapa 2 se definió luego de analizar los resultados de la etapa 1.

La selección de las estaciones se basó principalmente en el criterio de que estas mismas registraron datos durante todo el periodo definido para el procesamiento. Esto se logró analizando la información publicada en el sitio web www.epncb.oma.be. Previo a definir la estrategia de procesamiento, se hizo un listado de los receptores instalados en cada estación a procesar (ver tabla 2). Esto permitió determinar el tipo de observables que se recolecta en cada estación, debido a que se requiere conocer esta información para determinar la estrategia para fijar las ambigüedades (ver tabla 3 ).

Tabla 2

Registro del código P en L1 y L2 para las estaciones a procesar por sistema

\begin{tabular}{|c|c|c|c|c|}
\hline \multirow{2}{*}{ Estación } & \multirow{2}{*}{ Domes } & \multirow{2}{*}{ Tipo de receptor } & \multicolumn{2}{|c|}{ ¿Registra código $\mathrm{P}$ en $\mathrm{L} 1 / \mathrm{L} 2 ?$} \\
\hline & & & GPS & GLONASS \\
\hline AJAC & 10077M005 & LEICA GR25 & NO & NO \\
\hline BRST & $10004 \mathrm{M} 004$ & TRIMBLE NETR9 & NO & SÍ \\
\hline BUCU & $11401 \mathrm{M} 001$ & LEICA GRX1200GGPRO & NO & NO \\
\hline DYNG & $12602 \mathrm{M} 006$ & TRIMBLE NETR9 & NO & Sí \\
\hline \multirow{2}{*}{ FFMJ } & \multirow{2}{*}{ 14279M001 } & JPS LEGACY & Sí & Sí \\
\hline & & JAVAD TRE_G3TH DELTA & SÍ & SÍ \\
\hline GLSV & $12356 \mathrm{M} 001$ & NOV OEMV3 & NO & NO \\
\hline GOPE & 11502M002 & TPS NETG3 & Sí & Sí \\
\hline GRAS & 10002M006 & TRIMBLE NETR5 & NO & SÍ \\
\hline GRAZ & $11001 \mathrm{M} 002$ & LEICA GRX1200+GNSS & NO & NO \\
\hline \multirow{2}{*}{ HUEG } & \multirow{2}{*}{$14280 \mathrm{M} 001$} & LEICA GR25 & NO & NO \\
\hline & & JAVAD TRE_G3TH DELTA & SÍ & SÍ \\
\hline
\end{tabular}

continúa 
UNICIENCIA Vol. 31, No. 2, pp. 1-17. Julio-diciembre, 2017.

ISSN Electrónico: 2215-3470

URL: www.revistas.una.ac.cr/uniciencia

DOI: $\underline{\text { htp://dx.doi.org/10.15359/ru.31-2.1 }}$

Email: revistauniciencia@una.cr

\begin{tabular}{|c|c|c|c|c|}
\hline \multirow{2}{*}{ Estación } & \multirow{2}{*}{ Domes } & \multirow{2}{*}{ Tipo de receptor } & \multicolumn{2}{|c|}{ ¿Registra código $P$ en L1/L2? } \\
\hline & & & GPS & GLONASS \\
\hline KURE & $10604 S 001$ & LEICA GRX1200GGPRO & NO & $\mathrm{NO}$ \\
\hline LAMA & 12209M001 & LEICA GRX1200+GNSS & NO & NO \\
\hline LIL2 & $10051 \mathrm{M} 003$ & LEICA GR25 & NO & NO \\
\hline MARS & 10073M008 & LEICA GR25 & NO & NO \\
\hline MAR6 & 10405M002 & JPS E_GGD & Sí & Sí \\
\hline MATE & 12734M008 & LEICA GRX1200GGPRO & NO & NO \\
\hline ONSA & 10402M004 & JPS E_GGD & Sí & Sí \\
\hline ORID & $15601 \mathrm{M} 001$ & LEICA GRX1200GGPRO & NO & NO \\
\hline PENC & 11206M006 & LEICA GRX1200GGPRO & NO & NO \\
\hline POTS & $14106 \mathrm{M} 003$ & JAVAD TRE_G3TH DELTA & Sí & Sí \\
\hline \multirow{2}{*}{ SVTL } & \multirow{2}{*}{ 12350M001 } & TPS NETG3 & Sí & SÍ \\
\hline & & JAVAD TRE_G3TH DELTA & Sí & Sí \\
\hline SWKI & $12228 \mathrm{M} 001$ & TRIMBLE NETR5 & NO & SÍ \\
\hline TLSE & 10003M009 & TRIMBLE NETR9 & NO & SÍ \\
\hline VILL & $13406 \mathrm{M} 001$ & SEPT POLARX4 & Sí & NO \\
\hline \multirow{2}{*}{ WTZR } & \multirow{2}{*}{$14201 \mathrm{M} 010$} & LEICA GRX1200+GNSS & NO & NO \\
\hline & & LEICA GR25 & $\mathrm{NO}$ & NO \\
\hline
\end{tabular}

Nota: Elaboración propia.

Se analizó la disponibilidad del código P para el procesamiento GNSS, debido a que algunas de las estrategias de cálculo disponibles en el programa de procesamiento lo requieren. En el caso del sistema GPS, este código está reservado para fines militares, por lo que se envía encriptado al aplicar la técnica conocida como Anti-Spoofing (Hofmmann-Wellenhof et al., 2008), por lo que pocos receptores están en capacidad de registrarlo.

En el caso de GLONASS, el código si está liberado para los sujetos usuarios civiles, sin embargo, no todos los receptores de las estaciones incluidas en la presente investigación lo registran. Como consecuencia de esto hay estrategias disponibles en Bernese que no pueden ser aplicadas, debido a que requieren la información del código $\mathrm{P}$, como, por ejemplo, la combinación Melbourne-Wubbena (conocida también como L6). Por lo anterior, se ven limitadas las opciones aplicables para el procesamiento de los datos GNSS (ver tabla 3).

Como se desprende de la información dada en la tabla 2, 10 receptores tienen la capacidad de registrar el código P en GPS, mientras que 14 lo registran en GLONASS.

Con respecto al procesamiento de los datos, se efectuó con la licencia del programa Bernese, propiedad del CNPDG (Centro Nacional de Procesamiento de Datos GNSS), en su versión 5.2. Para el procesamiento de los datos GPS y GNSS se creó una campaña que se identificó de la 
siguiente forma respectivamente: WWWWGPS y WWWWGNSS donde WWWW representa la semana GPS que se procesó.

Se aclara que, de acá en adelante, cuando se hace referencia a datos GNSS, significa que son observaciones GPS y GLONASS que fueron procesadas de forma conjunta.

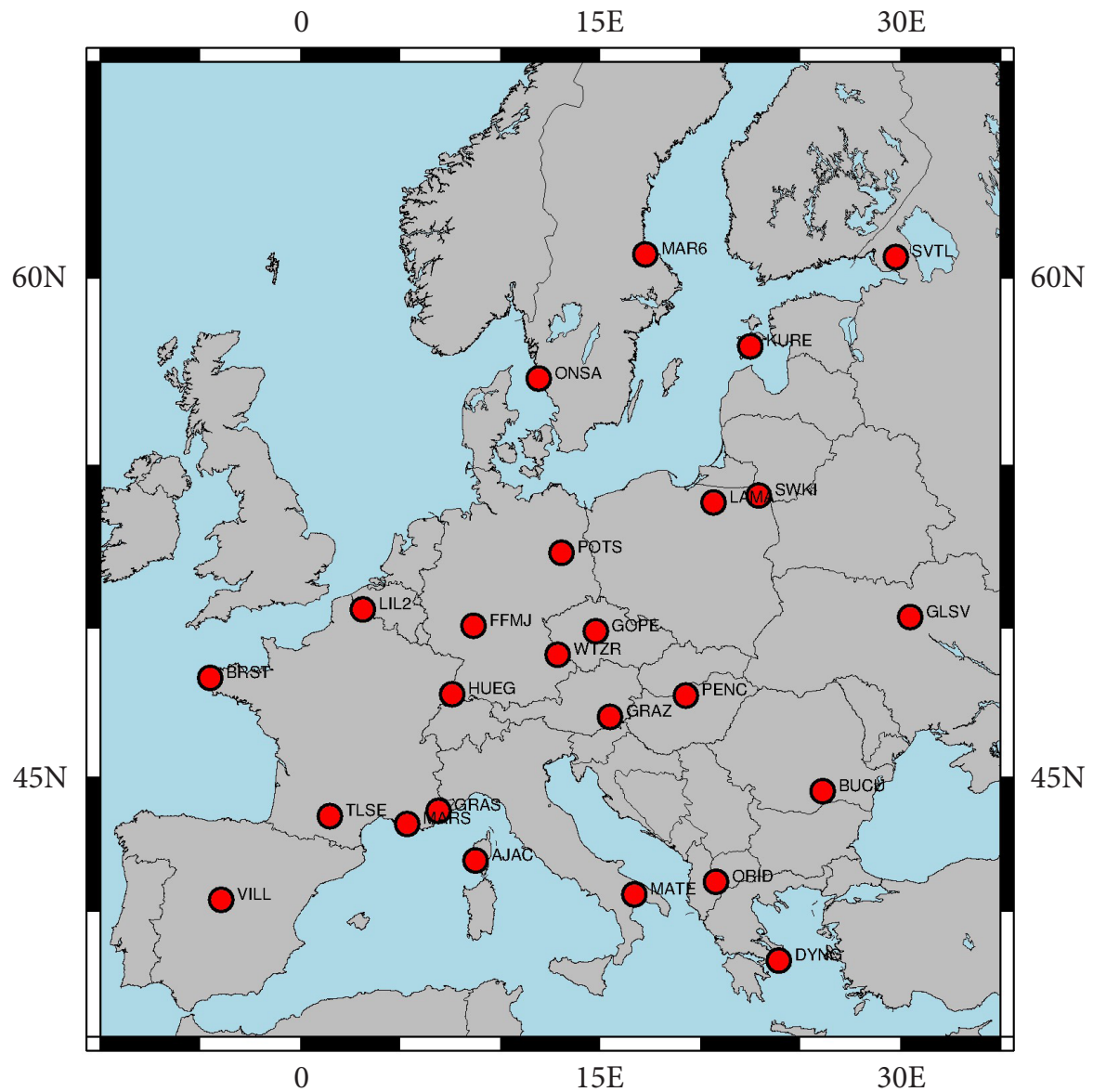

Figura 1. Diseño final de la red de estaciones de medición continua. Elaboración propia con base en datos del programa GMT.

Tabla 3

Estrategias utilizadas en el procesamiento

\begin{tabular}{lcc}
\hline \multicolumn{1}{c}{ Estrategias } & ¿Se utilizó? & Motivo de por qué no se usó \\
\hline SIGMA (L1 \& L2) & Sí & \\
SIGMA (MELWUEBB) & No & Procesa líneas base de hasta $6000 \mathrm{~km}$, sin embargo, se requiere el código P. \\
SEARCH & No & Procesa líneas base de hasta $10 \mathrm{~km}$ y no resuelve GLONASS. \\
ROUND & No & Procesa líneas base cortas, estrategia no recomendada. \\
QIF & Sí & \\
\hline
\end{tabular}

Nota: Elaboración propia. 
UNICIENCIA Vol. 31, No. 2, pp. 1-17. Julio-diciembre, 2017.

Email: revistauniciencia@una.cr

El procesamiento en las dos etapas se realizó con siguiente configuración:

- Intervalo de muestreo: 30 segundos

- Periodo de observación: diaria. Esto quiere decir que se dispone de un archivo de observación para cada estación, con registros de 24 horas

- Máscara de elevación: $3^{\circ}$

- Datos usados: datos del sistema GPS y GLONASS. Como requisito cada estación debe registrar señales de los dos sistemas

- Ponderación de las observaciones: se asignó un peso a las observaciones en función del ángulo de elevación del satélite; a menor elevación, menor peso

- Cálculos y presentación de resultados en tiempo GPS

- $\quad$ Modelo geoidal: EGM2008

- Las efemérides precisas y los parámetros de orientación de la Tierra (EOP’s, por sus siglas en inglés) usados en el procesamiento fueron los publicados por el IGS. Esta información se obtiene de la dirección $f t p: / / c d d i s . g s f c . n a s a . g o v / g p s /$ products

- Se usaron valores absolutos para las correcciones a las variaciones de los centros de fase de las antenas GNSS publicados por el IGS, incluyendo los valores dependientes de la elevación y azimut de entrada de la señal GNSS en la antena

- Para el modelado del efecto de la carga oceánica se usó el modelo de mareas oceánicas FES2004 (http://holt.oso.chalmers.se/loading/index.html)

- Para el modelado del efecto de la carga atmosférica se usó el modelo de componentes mareales S1 y S2, según el modelo Van-Dam y Rey (2010)

- Con respecto a la refracción troposférica, el retardo de la señal en el cenit de la estación se calculó simultáneamente con las coordenadas de la red, a un intervalo de una hora. El retardo a priori se estimó usando la función de mapeo de Viena (Vienna Mapping Function (VMF), por sus siglas en inglés), basado en las grillas globales publicadas por la Universidad Técnica de Viena (http://ggosatm.hg.tuwien.ac.at/DELAY/GRID/VMFG/)

- Para el modelo ionosférico se usaron los modelos globales publicados por el Centro Europeo para la Determinación de Orbitas (CODE, por sus siglas en inglés), disponibles en el sitio $\mathrm{ftp}: / / \mathrm{ftp}$.unibe.ch/aiub/CODE

- Selección de las líneas bases para el procesamiento: se usó el criterio de máximas observaciones, por lo que las líneas base procesadas son seleccionadas de forma automática por el programa basado en el criterio de la máxima cantidad de observaciones simultáneas entre dos estaciones

\section{Resultados del procesamiento de prueba (etapa 1)}

Como se comentó anteriormente, la etapa 1 de la investigación consistió en efectuar procesamientos de prueba modificando la estrategia para resolver ambigüedades. Debido a lo 
comentado sobre el requerimiento del código $\mathrm{P}$ en algunas estrategias de cálculo, se procesó con las estrategias QIF y SIGMA. En esta etapa se procesaron tres semanas GPS (1774, 1775 y 1776), con el fin de determinar la estrategia que resuelve el mayor porcentaje de ambigüedades; estos procesamientos se comentan a continuación:

1. Para el procesamiento de los datos GPS se utilizó la combinación de frecuencia L3 (libre de ionósfera) con dobles diferencias y se resolvieron las ambigüedades con la estrategia QIF. Los resultados de este procesamiento son considerados de referencia para efectuar el análisis de los resultados.

2. Usar la combinación de frecuencias L3, con dobles diferencias y resolver ambigüedades con la estrategia QIF. Esta estrategia se aplicó para el procesamiento de datos GNSS.

3. Utilizar la combinación de frecuencias L3, con dobles diferencias y resolver ambigüedades con la estrategia SIGMA. Esta estrategia se aplicó para el procesamiento de datos GNSS.

Luego de analizar los resultados se determinó que con la estrategia SIGMA no se estaban resolviendo las ambigüedades, por lo que se hizo la consulta a la Universidad de Berna del porqué sucedía esto y la respuesta fue que esta estrategia aplica para líneas base cortas que dispongan datos en L1 y L2 (líneas base cortas de 20 a $40 \mathrm{~km}$ ).

Por tanto, se utilizó QIF para el procesamiento de datos GPS y GNSS debido a que se considera que para los fines del proyecto es la mejor estrategia disponible en Bernese, ya que trabaja directamente sobre las portadoras L1 y L2 de ambos sistemas y resuelve ambigüedades y líneas base de hasta $2000 \mathrm{Km}$.

La figura 2 muestra los resultados con el porcentaje de ambigüedades resueltas para el procesamiento GPS y GNSS. Se observan dos líneas para el procesamiento GNSS debido a que, si bien es cierto el procesamiento combina datos GPS y GLONASS, la resolución de ambigüedades se hace sobre las portadoras de cada sistema de forma independiente (esto es válido para las figuras donde se muestra la comparación de resultados entre los procesamientos).

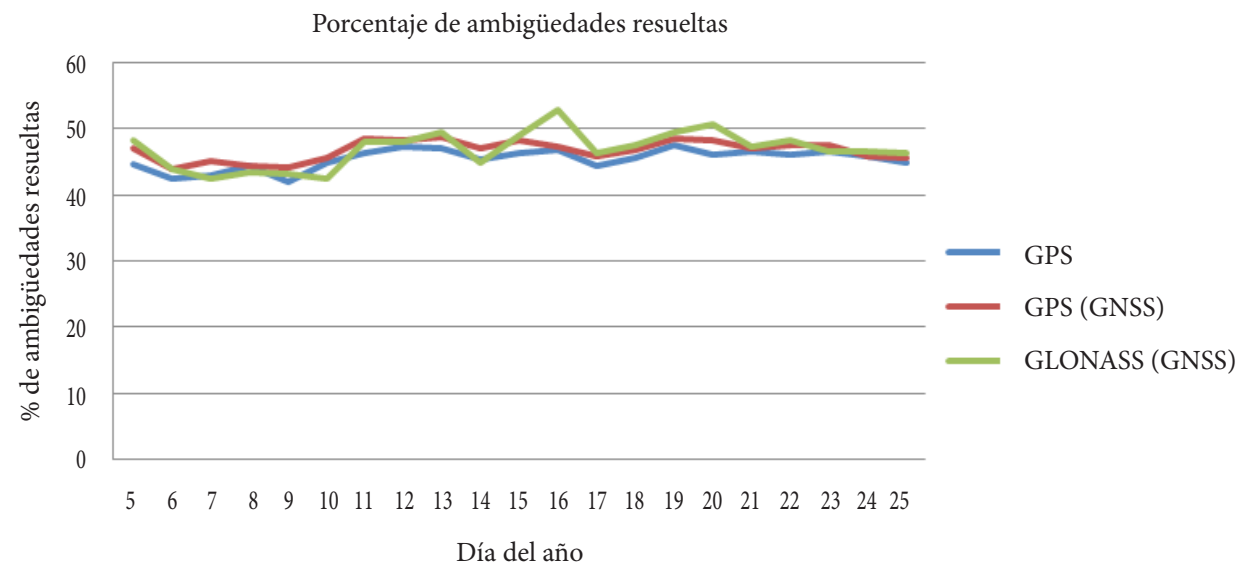

Figura 2. Gráfica con porcentaje de ambigüedades resueltas en el procesamiento de datos GPS y GNSS. Elaboración propia del estudio. 
UNICIENCIA Vol. 31, No. 2, pp. 1-17. Julio-diciembre, 2017.

Email: revistauniciencia@una.cr

Luego del procesamiento de datos GPS y GNSS, ambos con la estrategia QIF, se determinó que no hay incremento notorio en el porcentaje de ambigüedades resueltas; entre el procesamiento GPS y GNSS, ambos con la estrategia QIF, el porcentaje de ambigüedades resueltas es menor al $50 \%$. Por esto, el procesamiento de ambos conjuntos de datos se realizó con la estrategia QIF.

\section{Resultados del procesamiento para el periodo completo (etapa 2)}

La etapa 2 consistió en procesar 26 semanas del año 2014, iniciando en la semana GPS 1774. Con base en los resultados de la etapa 1 los datos fueron procesados con la estrategia QIF.

El primer análisis sobre el porcentaje de ambigüedades resueltas se basó en el uso de histogramas, ya que con este tipo de gráfico resulta sencillo determinar la frecuencia con la que se presentan los diferentes resultados. Para generarlos se realizó una rutina en $\mathrm{Matlab}^{\circledR}$, para la cual se tabuló el promedio para cada día del periodo de procesamiento. Esto mismo se hizo para dos líneas base en particular: SWKI-WTZR por ser la que presenta un menor porcentaje de ambigüedades resueltas (en promedio 30\%) y SVTL-SWKI debido a que es la que presenta un mayor porcentaje (en promedio 50\%).

En la figura 3 se observa el histograma del porcentaje de ambigüedades resueltas para el procesamiento de únicamente datos GPS por día; en la figura 4 se muestra el resultado del promedio diario de las ambigüedades resueltas para el procesamiento de solo datos GPS.

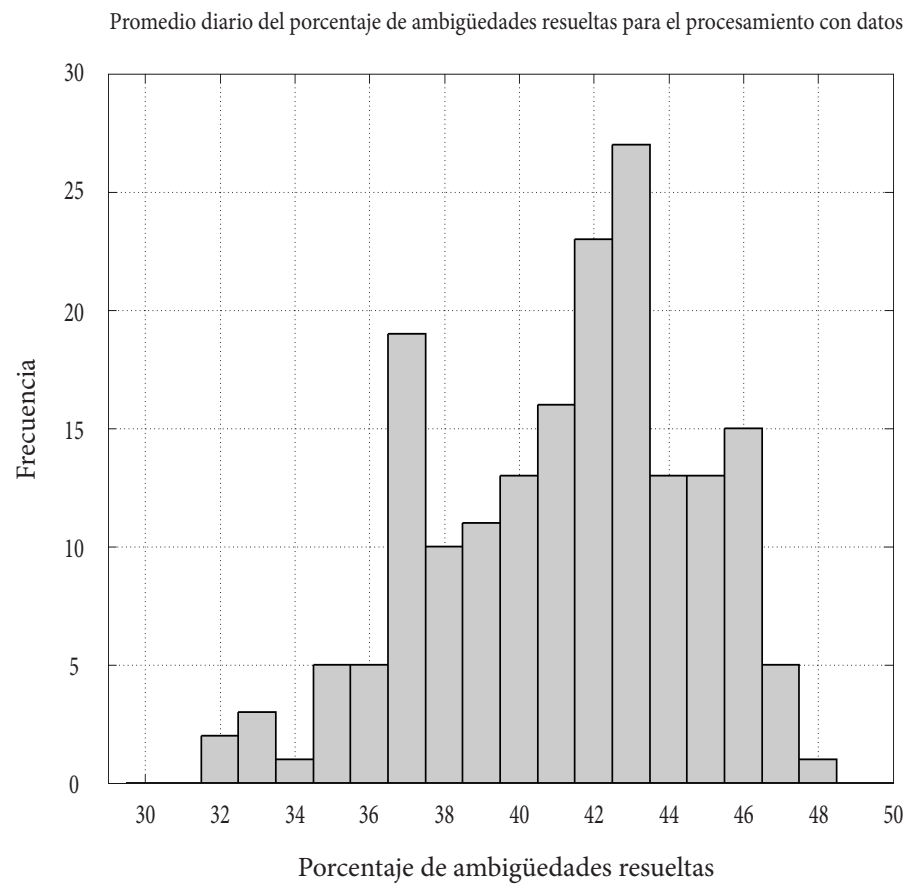

Figura 3. Histograma del porcentaje de ambigüedades resueltas del promedio diario. Elaboración propia. 
De la figura 4 se aprecia que el porcentaje de ambigüedades resueltas para los datos GPS presenta tres periodos con comportamientos diferentes; en primera instancia entre los días 005 y 089 es en promedio de entre 40\% y 48\%; entre los días 090 y 119 se observa una disminución, que varía entre el 33\% y el 42\% y finalmente a partir del día 120 y hasta el día 186, el porcentaje se incrementa con algunas fluctuaciones.

La línea SVTL-SWKI presenta una particularidad: desde el día del año 005 hasta el 091, el porcentaje de ambigüedades resueltas era uno de los más altos, sin embargo, el día 092 hubo un cambio en el receptor de la estación SVTL, pasando de un Topcon TPS NETG3 a un JAVAD TRE_G3TH DELTA, lo cual se cree que provocó que la cantidad a partir del día 093 fuera mucho menor, esto se puede apreciar en la figura 5, donde es evidente la disminución.

En la figura 6 se aprecia que el porcentaje de ambigüedades resueltas GLONASS para la línea SVTL-SWKI antes del día 091 tiene un comportamiento distinto al de la señal GPS, incluso tiene un porcentaje menor al del procesamiento GPS, sin embargo, después de este día su comportamiento es semejante al de GPS. Se piensa que el motivo del descenso fue el cambio a otro equipo, ya que la estación WTZR también sufrió un cambio en el receptor, pero este fue entre la misma casa fabricante, en este caso LEICA.

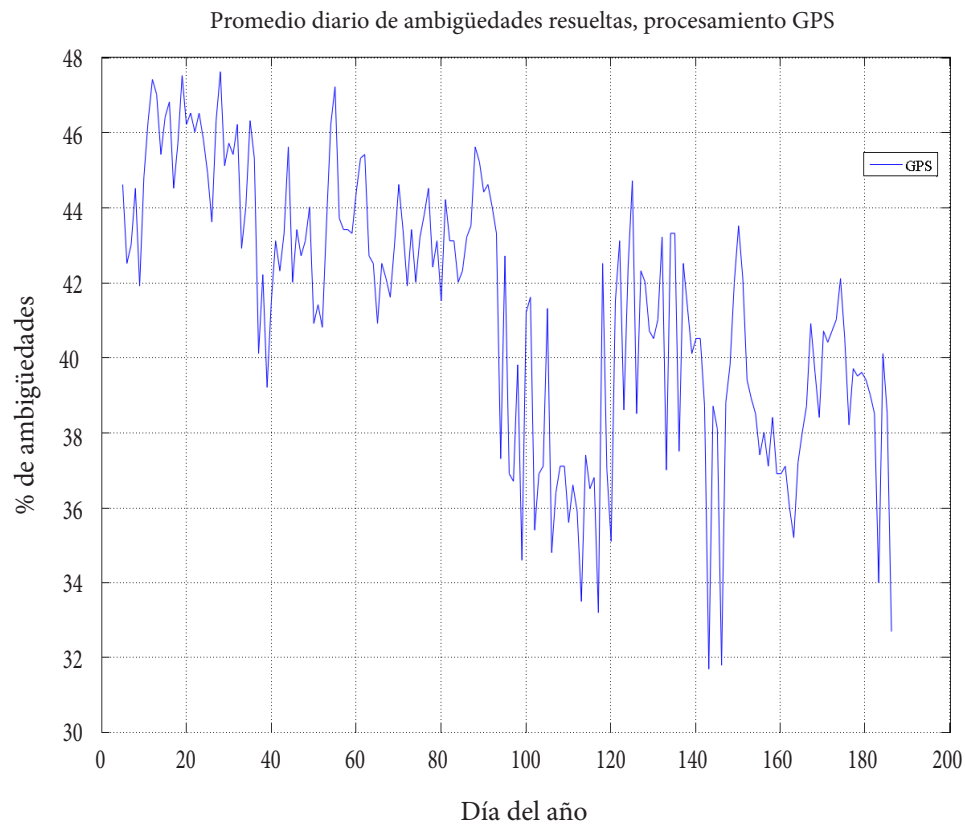

Figura 4. Porcentaje de ambigüedades resueltas del promedio diario. Elaboración propia. 
UNICIENCIA Vol. 31, No. 2, pp. 1-17. Julio-diciembre, 2017.

ISSN Electrónico: 2215-3470

URL: www.revistas.una.ac.cr/uniciencia

DOI: http://dx.doi.org/10.15359/ru.31-2.1

Email: revistauniciencia@una.cr

Porcentaje de ambigüedades resueltas por día para la línea base SVTL-SWKI, procesamiento GPS

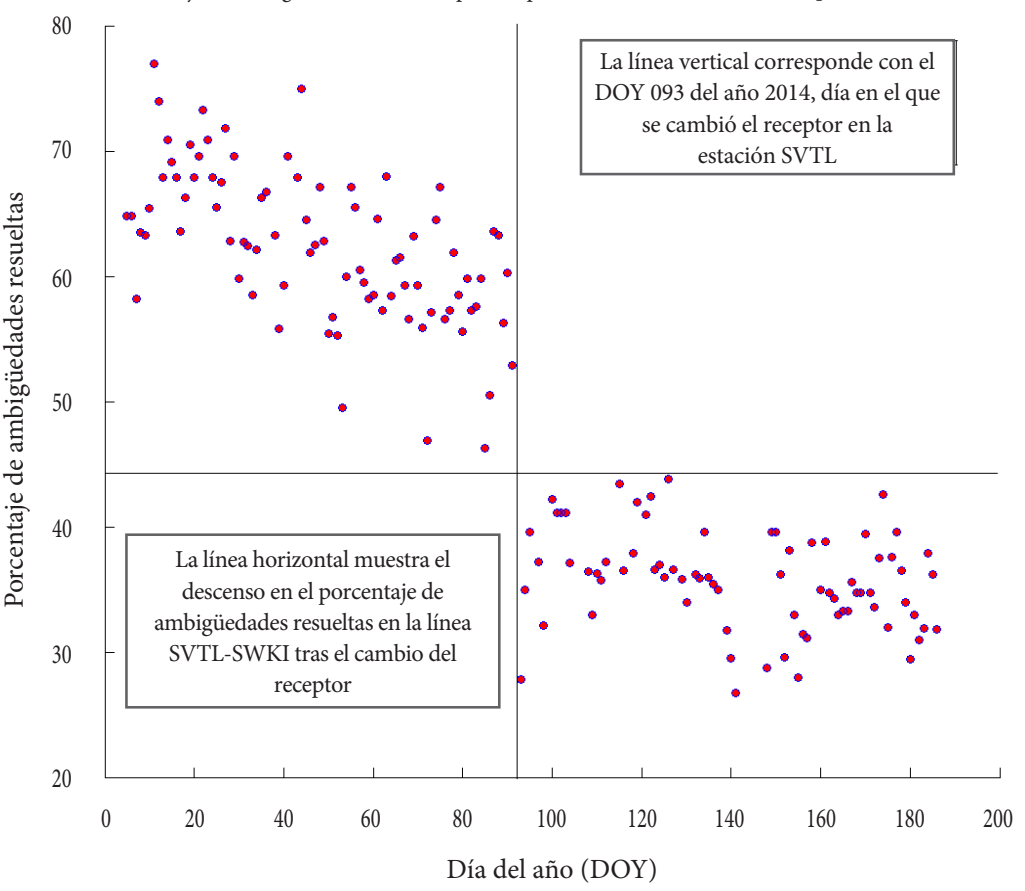

Figura 5. Cambio en el receptor línea SWKI-SVTL. Elaboración propia.

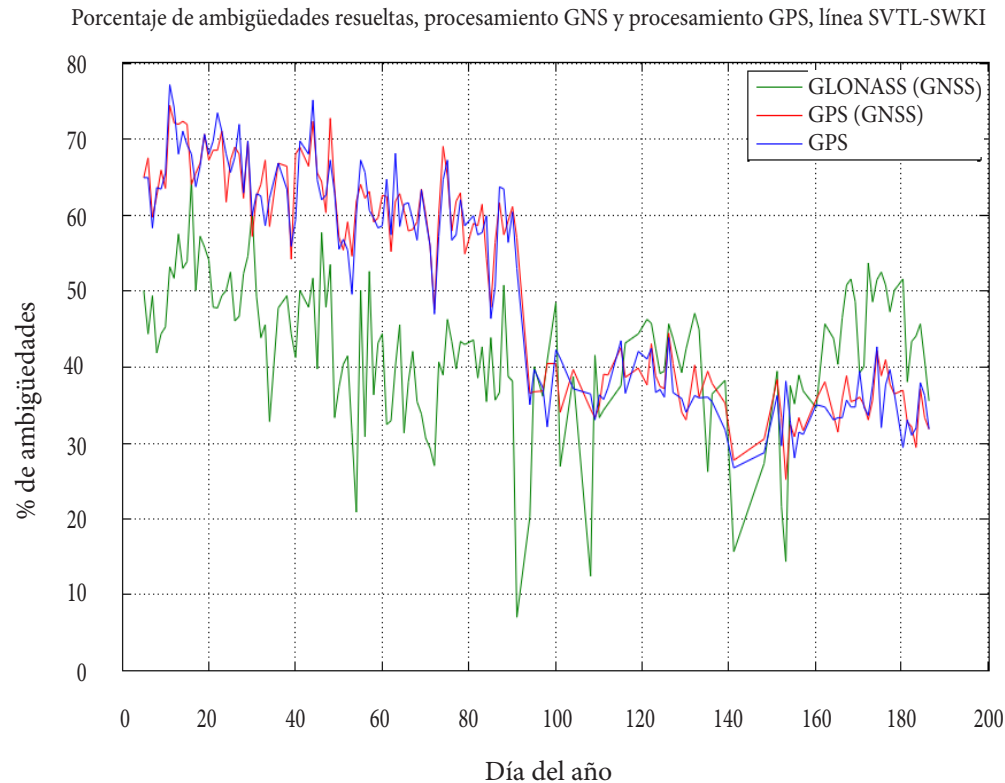

Figura 6. Comparación entre procesamientos del porcentaje de ambigüedades resueltas, línea SVTL-SWKI. Elaboración propia. 
En la figura 7 se observa cómo el porcentaje de ambigüedades resueltas para los datos GPS (tras ambos procesamientos) tiene un comportamiento similar para todos los días, sin embargo, se contempla que el porcentaje en GLONASS a partir del día 120 tiende a ser mayor que el de GPS, a pesar de fluctuaciones específicas como la del día 145.

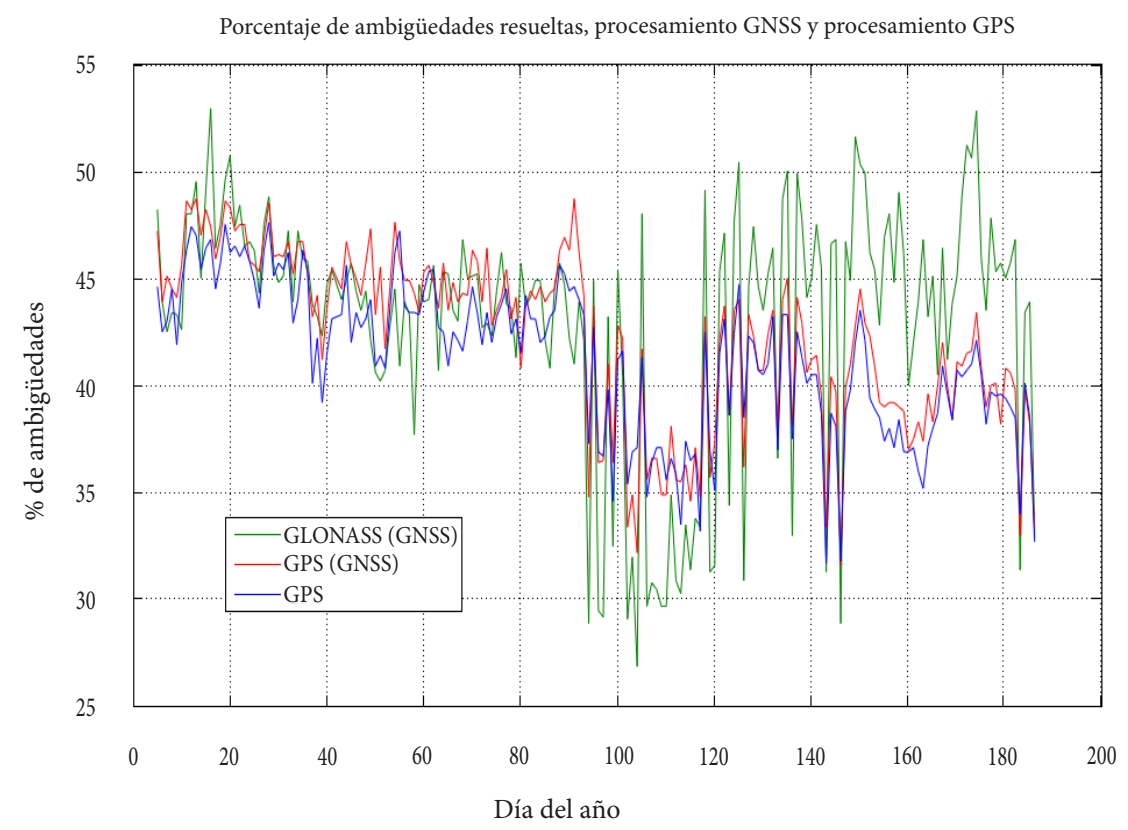

Figura 7. Porcentaje de ambigüedades resueltas, GNSS vs GPS. Elaboración propia.

En general y luego del análisis de resultados obtenidos en el procesamiento de la etapa 2 (186 días procesados) se determina que el promedio diario de las ambigüedades resueltas para los procesamientos GPS y GNSS, utilizando la estrategia QIF, no cambia notoriamente, ya que en promedio el porcentaje de ambigüedades resueltas es de $41 \%$ para GPS y $43 \%$ para GNSS (GPS y GLONASS).

El porcentaje de ambigüedades resueltas para ambos procesamientos es menor al esperado, en promedio es inferior al 50\%. Esto no tiene una gran influencia en la estimación de otros parámetros como el retardo troposférico cenital; no obstante, sí tiene implicaciones en el tiempo invertido por el programa para efectuar el ajuste final de la red y en las capacidades de hardware requeridas para la computadora donde está instalado el programa, debido a que se requiere mayor capacidad de memoria para poder efectuar el procesamiento de los datos.

No se logró determinar la causa de lo comentado sobre la figura 7, donde se observa que luego del día 120, la cantidad de ambigüedades resueltas para el sistema GLONASS es mayor que para GPS (en ambos procesamientos donde se trabajó con las señales de este sistema). Es posible que haya una influencia relacionado con la geometría de la red e incluso aspectos vinculados directamente con los modelos matemáticos que el programa aplica para el procesamiento de los datos. 
UNICIENCIA Vol. 31, No. 2, pp. 1-17. Julio-diciembre, 2017.

ISSN Electrónico: 2215-3470

URL: www.revistas.una.ac.cr/uniciencia

DOI: http://dx.doi.org/10.15359/ru.31-2.1

\section{Conclusiones}

Para determinar la estrategia con la cual se procesarían los datos GNSS se analizó la disponibilidad del código P, el cual está liberado en el sistema GLONASS. Sin embargo, no todos los receptores de las estaciones utilizadas en la red del proyecto lo registran. Por esta razón, cualquier estrategia que requiriera del código $\mathrm{P}$ no pudo ser evaluada.

Relacionado con el tema de resolución de ambigüedades se descartaron las estrategias SEARCH y ROUND, ya que SEARCH no resuelve ambigüedades GLONASS y ROUND se aplica en líneas base cortas y por su naturaleza no utiliza la información de la varianza-covarianza.

Se realizó un procesamiento de prueba con el fin de determinar la estrategia de procesamiento de los datos GNSS. Esta prueba consistió en procesar tres semanas del de datos $(1774,1775$ y 1776) utilizando la estrategia QIF y SIGMA.

Se usó la estrategia QIF para el procesamiento de los datos GPS y GNSS, ya que esta estrategia trabaja directamente sobre las portadoras L1 y L2, con la ventaja de que esta resuelve ambigüedades del sistema GLONASS.

También se usó la estrategia SIGMA para el procesamiento GNSS, pero no se siguió procesando con esta, ya que esta resuelve las ambigüedades para líneas base menores a $40 \mathrm{~km}$ con la combinación lineal L1\&L2 y en el presente proyecto las longitudes de las líneas base de la red son en promedio de $600 \mathrm{Km}$.

No se pudieron implementar otras estrategias de procesamiento, debido a que estas no se pueden aplicar con los datos registrados por las estaciones utilizadas.

Tras comparar el promedio diario de las ambigüedades resueltas para los procesamientos GPS y GNSS, ambos con la estrategia QIF, se concluye que no hay incremento notorio en este, ya que en promedio el porcentaje de ambigüedades resueltas es de $41 \%$ para GPS y $43 \%$ para el procesamiento GNSS (datos GPS y GLONAS procesados de manera conjunta), desde la consideración de que la totalidad de los receptores utilizados registra datos de los sistemas GPS y GLONASS.

El porcentaje de ambigüedades resueltas para ambos procesamientos es menor al esperado, ya que en general este es inferior al 50\%. Esto no influye directamente sobre los resultados del procesamiento de la red, aunque sí tiene un impacto al incrementarse la cantidad de ambigüedades a estimar.

\section{Referencias}

Bruyninx, C. (2006). Comparing GPS-only with GPS + GLONASS positioning in a regional permanent GNSS network. GPS Solutions, 11(2), 97-106. http://dx.doi.org/10.1007/s10291-006-0041-9

Cioce, V., Robin, A., Mateo, L., \& Mackern, V. (2013). Avances en la incorporación de observaciones GLONASS al ajuste de la red SIRGAS-CON [Presentación de Power Point]. Panamá: SIRGAS. 13 diapositivas.

Dach, R., \& Walser, P. (2013). Bernese GNSS Software Version 5.2. Course Tutorial. http://www.bernese. unibe.ch/docs/TUTORIAL.pdf

Dach, R., Lutz, S., Walser, P., \& Fridez, P. (2015). Bernese GNSS Software Version 5.2. Berna: Publikation Digital AG.

Habrich, H. (2009). H.Drewes (ed.), Geodetic Reference Frames, International Association of Geodesy Symposia 134, DOI 10.1007/978-3-642-00860-3_19, ® Springer-Verlag Berlin Heidelberg 2009. 
ISSN Electrónico: 2215-3470

DOI: http://dx.doi.org/10.15359/ru.31-2.1
UNICIENCIA Vol. 31, No. 2, pp. 1-17. Julio-diciembre, 2017.

URL: www.revistas.una.ac.cr/uniciencia Email: revistauniciencia@una.cr

Hofmann - Wellenhof, B., Lichtenegger, H., \& Wasle, E. (2008). GNSS - Global Navigation Satellite Systems. Austria: Springer-Verlag Wien.

IGS Central Bureau. (8 de abril de 2004). IGS Central Bureau Information System. Recuperado de IGS Central Bureau Information System: http://www.igs.org/overview/viewindex.html

Mervart, L. (1995), Ambiguity Resolution Techniques in Geodetic and Geodynamic Applications of the Global Positioning System. Suiza: Geodätisch-geophysikalische Arbeiten in der Schweiz.

Van-Dam, T., \& Ray, R. (2010). S1 and S2 Atmospheric Tide Loading Effects for Geodetic Applications. Data set/Moddel at http://geophy.uni.lu/ggfc-atmosphere/tide-loading-calculator.html

Willis, P., Slater, J., Gurtner, W., Noll, C., Beutler, G., Weber, R., \& Hein, G. (1999). The GLONASS IGEX-98 Campaign: From Its Genesis to Its Realization. Tennessee: California Institute of Technology.

$\mathrm{Xu}, \mathrm{G}$. (2007). GPS Theory, Algorithms and Applications. Alemania: Springer-Verlag Berlin Heidelberg.

\section{(c) (i) (9)}

Comparación de los resultados en la resolución de ambigüedades para una red de estaciones GNSS en Europa usando datos GPS y la combinación con datos GLONASS, utilizando el programa de procesamiento Bernese (Diana Paniagua-Jiménez y otros) por Revista Uniciencia se encuentra bajo una Licencia CreativeCommons Atribución-NoComercial-SinDerivadas 3.0

Unported 